\title{
Mitral valve annuloplasty and anterior leaflet augmentation for functional ischemic mitral regurgitation: Quantitative comparison of coaptation and subvalvular tethering
}

\author{
Jean-Pierre M. Rabbah, PhD, ${ }^{a}$ Andrew W. Siefert, $\mathrm{PhD},{ }^{\mathrm{a}}$ Steven F. Bolling, MD, ${ }^{\mathrm{b}}$ and \\ Ajit P. Yoganathan, $\mathrm{PhD}^{\mathrm{a}}$
}

\begin{abstract}
Objective: Although restrictive mitral annuloplasty (RMA) has been the preferred surgical treatment of functional ischemic mitral regurgitation (FIMR), some patients with severely dilated left ventricles will experience recurrent mitral regurgitation (MR). Consequently, new surgical strategies have been entertained to compensate for severely dilated ventricles by maximizing coaptation and reducing subvalvular tethering. Anterior leaflet augmentation (ALA) with mitral annuloplasty has been theorized to meet these goals. We compared the mechanistic effects of RMA and adjunct ALA in the setting of FIMR.
\end{abstract}

\begin{abstract}
Methods: Mitral valves were mounted in a clinically relevant left heart simulator. The tested conditions included control, FIMR, RMA, and true-size annuloplasty with either a small or large ALA. The A2-P2 leaflet coaptation length, MR, and strut and intermediary chordal forces were quantified. All repairs alleviated the MR. The coaptation length was significantly increased from FIMR to RMA, small ALA, and large ALA $(P<.001)$. Between repairs, a large ALA created the greatest length of coaptation $(P<.05)$. Tethering forces from the posteromedial strut chordae were reduced from the FIMR condition by all repairs $(P<.001)$. Only a large ALA reduced the intermediate chordal tethering from the FIMR condition $(P<.05)$.

Conclusions: A large ALA procedure created the greatest coaptation and reduced chordal tethering. Although all repairs abolished MR acutely, the repairs that create the greatest coaptation might conceivably produce a more robust and lasting repair in the chronic stage. A clinical need still exists to best identify which patients with altered mitral valve geometries would most benefit from an adjunct procedure or RMA alone. (J Thorac Cardiovasc Surg 2014;148:1688-93)
\end{abstract}

Functional ischemic mitral regurgitation (FIMR) has been classified as a Carpentier type IIIb valvular dysfunction that results from postinfarction left ventricular (LV) remodeling. ${ }^{1}$ This disease poses a significant clinical challenge, because FIMR severity is directly associated with patient mortality. ${ }^{2}$ Although significant knowledge of FIMR and its physiologic effect exists, the surgical management of FIMR remains controversial. ${ }^{3-5}$ Amid this controversy, restrictive mitral annuloplasty (RMA) with a complete rigid ring has been regarded as a preferred surgical

From the Wallace H. Coulter Department of Biomedical Engineering, ${ }^{a}$ Georgia Institute of Technology and Emory University, Atlanta, Ga; Department of Cardiac Surgery, ${ }^{\mathrm{b}}$ Cardiovascular Center, University of Michigan Hospitals, Ann Arbor, Mich.

The present study was partially supported by a research grant from the National Institutes of Health (R01 HL113216).

Disclosures: Steven F. Bolling reports consulting fees from Edwards and Medtronic and grant support from Edwards and Abbott. All other authors have nothing to disclose with regard to commercial support.

Drs J.-P.M.R. and A.W.S. contributed equally to the present study.

Received for publication Dec 23, 2013; revisions received March 20, 2014; accepted for publication April 4, 2014; available ahead of print June 3, 2014.

Address for reprints: Ajit P. Yoganathan, PhD, Wallace H. Coulter Department of Biomedical Engineering, Georgia Institute of Technology, 313 Ferst Dr, Suite 2119, Atlanta, GA 30332-0535 (E-mail: ajit.yoganathan@bme.gatech.edu).

$0022-5223 / \$ 36.00$

Copyright (c) 2014 by The American Association for Thoracic Surgery

http://dx.doi.org/10.1016/j.jtcvs.2014.04.008 repair. ${ }^{3,6}$ Although typically effective, approximately $10 \%$ to $15 \%$ of patients presenting with severe LV dilation will develop recurrent mitral regurgitation (MR), have low rates of reverse LV remodeling, and exhibit poor survival. ${ }^{7-10}$

Consequently, new adjunct surgical strategies have been attempted to compensate for severely dilated left ventricles. ${ }^{4}$ When performed with mitral annuloplasty, these adjunct strategies have aimed to further alleviate chordal leaflet tethering, improve leaflet mobility, and maximize leaflet coaptation. Anterior leaflet augmentation (ALA) $)^{5,11}$ has been recently demonstrated to restore leaflet coaptation and mobility without the need to unnaturally restrict the mitral annulus. ${ }^{10,11}$ Although preliminary studies have demonstrated the potential benefits of both RMA and ALA, little knowledge exists of what mechanistic improvements in valve function can be expected.

In the present study, we aimed to elucidate the functional and mechanical benefits of ALA with true-size mitral annuloplasty compared with isolated RMA in the setting of FIMR. ALA was performed with 2 pericardial patch sizes to further elucidate the potential effects of the procedural technique. We hypothesized that ALA will achieve leaflet coaptation comparable to that of RMA, while yielding significant improvements in leaflet tethering as measured by chordal forces. 


\section{Abbreviations and Acronyms \\ ALA $=$ anterior leaflet augmentation \\ FIMR $=$ functional ischemic mitral regurgitation \\ $\mathrm{LV}=$ left ventricular \\ MR $=$ mitral regurgitation \\ MV = mitral valve \\ $\mathrm{PM}=$ papillary muscle \\ RMA $=$ restrictive mitral annuloplasty}

\section{METHODS}

\section{In Vitro Simulator}

The extensively studied Georgia Tech left heart simulator was used for the present study. ${ }^{12}$ This in vitro model has been previously demonstrated to mimic the systolic mitral valve (MV) geometry, ${ }^{12}$ leaflet coaptation, regurgitation, and anterior leaflet strain of healthy and chronic FIMR ovine models. In this model, ovine MVs were excised, mounted into the simulator, and tested using pulsatile left side heart hemodynamics.

\section{Chordal Force Transducers}

Miniature C-shaped force transducers have been used previously to quantify the tethering forces of the MV's chordae tendineae. ${ }^{13}$ These strain gauge-based transducers were manufactured and tested within our laboratory. Calibration was performed before and after each experiment. The relationship between the calibrated load and transducer voltage output was linear, with a regression coefficient $\left(\mathrm{R}^{2}\right)$ of 0.98 to 1.00 . The relative difference between the measured and true calibrated values (accuracy) was $<2 \%$. The smallest measurable tension was $0.01 \mathrm{~N}$. ${ }^{13}$ Before each experiment, these transducers were sutured directly to selected chordae without altering the chordae's native length. The section of chordae located between the transducer's measurement arms was bisected such that all tensile loading of the chord was transferred to the transducer.

\section{Experimental Preparation}

For the present study, fresh ovine hearts $(\mathrm{n}=15)$ were procured from a local abattoir. The MVs were excised, preserving their annular and subvalvular anatomy. MVs with an anterior leaflet height of 20 to $25 \mathrm{~mm}$, type I or II papillary muscles (PMs), and all leaflet chordae inserting directly into each PM were selected for experimentation. Selected MVs were sutured to the simulator's annulus using a Ford interlocking stitch. During MV suturing, care was taken to place each suture just above the MV's natural hinge and not through the leaflet tissue. Additionally, the normal annular-leaflet geometric relationships were respected, with the anterior leaflet occupying one third of the annular circumference and commissures aligned in the 2- and 10-o'clock positions. After annular suturing, the strut chordae inserting into the anterior leaflet $(\mathrm{n}=2)$ and intermediary chordae inserting into the posterior leaflet $(\mathrm{n}=2)$ were instrumented with chordal force transducers. After instrumentation, each PM was attached to the positioning control rods.

\section{Experimental Protocol}

After mounting each of the instrumented MVs within the simulator, the mitral annulus was conformed to the shape of a size 30 Physio Annuloplasty Ring (Edwards Lifesciences, Irvine, Calif). On establishing human pulsatile left heart hemodynamics (cardiac output, $5.0 \mathrm{~L} / \mathrm{min}$; heart rate, $70 \mathrm{bpm}$; transmitral pressure, $120 \mathrm{~mm} \mathrm{Hg}$ ), each PM was carefully positioned to establish the control MV geometry. ${ }^{14}$ Mitral coaptation was inspected using echocardiography. The anterior leaflet spanned two thirds of the A2-P2 annular diameter, 5 to $6 \mathrm{~mm}$ of coaptation was achieved, and $<1 \mathrm{~mm}$ tenting was observe. If the control valve geometry conditions had been successfully achieved, transmitral flow, left atrial and ventricular pressure, 3-dimensional echocardiography (Philips iE-33 Matrix, Phillips Healthcare, Andover, Mass), Doppler echocardiography, and chordal forces were acquired.

To simulate chronic FIMR due to inferior myocardial infarction, the valve annulus was asymmetrically dilated to $150 \%$ of the control valve area. The anterolateral PM was displaced $3 \mathrm{~mm}$ apically and $2 \mathrm{~mm}$ anteriorly, and the posteromedial PM was displaced $4 \mathrm{~mm}$ apically, $4 \mathrm{~mm}$ posteriorly, and $8 \mathrm{~mm}$ laterally. These changes were consistent with the data from chronic FIMR due to inferior myocardial infarction. ${ }^{1}$ After establishing these conditions, all experimental measurements were acquired.

In the present study, 3 repair strategies were evaluated, including restrictive mitral annuloplasty ( $\mathrm{n}=15)$, true-size annuloplasty with a "small" ALA $(\mathrm{n}=8)$, and true-size annuloplasty with a "large" ALA $(\mathrm{n}=7)$. Restrictive and true-size mitral annuloplasty were simulated by conforming the simulator's annulus to a size 26 and size 30 Physio ring, respectively (Figure 1).

The ALAs were randomized and completed in 2 sizes using bovine pericardium lightly fixed in $0.5 \%$ glutaraldehyde. For the large ALA, the patch dimensions were $2.68 \pm 0.25 \mathrm{~cm}$ long, $1.07 \pm 0.10 \mathrm{~cm}$ high, and an area of $2.26 \pm 0.36 \mathrm{~cm}^{2}$. For the small ALA, the patch dimensions were $1.77 \pm 0.10 \mathrm{~cm}$ long, $0.65 \pm 0.14 \mathrm{~cm}$ high, and an area of $0.86 \pm 0.29 \mathrm{~cm}^{2}$. An incision was made near the base of the anterior leaflet and parallel to the mitral annulus. This created an oblong-shaped opening in the anterior leaflet to which the patch was sutured. The length of the incisions corresponded to the length of the desired patch dimensions. With either ALA repair, a concomitant true-size annuloplasty procedure was performed. The pericardial patch was positioned in place using 4 interrupted knots in the 3-, 6-, 9-, and 12-o'clock positions. A running mattress suture was used to secure the pericardium to the leaflet and create a leakfree seal.

\section{Statistical Analysis}

All hemodynamic and chordal force data were ensemble averaged for 10 cardiac cycles. MR was quantified by integrating the negative (or reverse) flow over the systolic portion of the cardiac cycle. The presence of MR was confirmed by Doppler echocardiography. Philips Qlab (Phillips Healthcare, Andover, Mass) was used to quantify the MV leaflet coaptation length measured at the A2-P2 coaptation line. Chordal force data are presented as the difference between the peak systolic and peak diastolic force. The percentage of improvement in coaptation length (increase in coaptation length) and chordal force (reduction in force) was calculated between the postoperative repair and FIMR groups.

The measured endpoints were checked for normality using the Anderson-Darling test. The independent samples $t$ test and 2-way analysis of variance were used when appropriate. A Tukey post hoc test was used to further investigate significance. All statistical analyses were completed using Minitab, version 16 (Minitab, Inc, State College, Pa). All data are reported as the mean \pm standard deviation.

\section{RESULTS \\ Functional Ischemic MR}

FIMR was successfully simulated with asymmetric annular dilatation and PM displacement for all experiments ( $3+$ MR grade, $n=4 ; 4+$ MR grade, $n=11)$. Large, asymmetric regurgitant jets were observed using color Doppler echocardiography. Leaflet tethering was observed, and the coaptation length was significantly decreased from that of the control group (control group, $4.8 \pm 0.5 \mathrm{~mm}$; FIMR group, $3.2 \pm 0.5 \mathrm{~mm} ; P<.005)$ at the central A2-P2 plane (Table 1). 


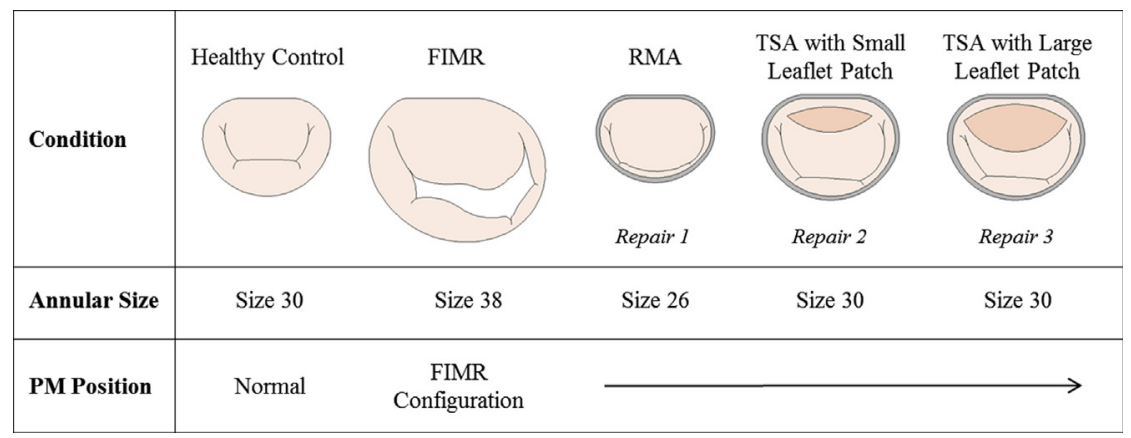

FIGURE 1. Experimental conditions (annulus and anterior leaflet patch sizes) are shown in this schematic representation of the experimental protocol. FIMR, Functional ischemic mitral regurgitation; RMA, restrictive mitral annuloplasty; TSA, true-size annuloplasty; PM, papillary muscle.

For the posteromedial PM, the tethering force in the anterior strut chordae was significantly increased compared with that of the control group (control group, $0.71 \pm 0.31 \mathrm{~N}$; FIMR group, $1.23 \pm 0.44 \mathrm{~N} ; P=.001$; Table 1 and Figure 2). Similarly, the force in the posterior intermediate chordae was significantly increased compared with that in the control group (control group, $0.28 \pm 0.13 \mathrm{~N}$; FIMR group, $0.55 \pm 0.29 \mathrm{~N} ; P=.006$; Table 1 and Figure 2).

No statistically significant changes between the control and FIMR conditions were observed for the anterior strut (control group, $0.62 \pm 0.44 \mathrm{~N}$; FIMR group, $0.80 \pm$ $0.52 \mathrm{~N}$ ) and posterior intermediate chordae (control group, $0.49 \pm 0.34 \mathrm{~N}$; FIMR group, $0.71 \pm 0.51 \mathrm{~N}$ ) originating from the anterolateral PM (Table 1 and Figure 2).

\section{Restrictive Annuloplasty Versus ALA With True-Size Annuloplasty}

All surgical repairs alleviated MR, and no regurgitant jets were observed postoperatively. Additionally, all repairs significantly $(P<.005)$ increased the coaptation length from FIMR back to, or greater than, healthy levels (Table 1 and Figure 3). The large patch procedure with true-size annuloplasty resulted in the best improvement in coaptation length $(98.3 \% \pm 31.0 \%$ increase) compared with FIMR (Figure 3). Restrictive mitral annuloplasty
$(51.0 \% \pm 21.3 \%$ increase $)$ and the small patch with truesize annuloplasty $(43.2 \% \pm 16.5 \%$ increase $)$ resulted in comparable improvement in the coaptation length compared with FIMR (Figure 3). Furthermore, the postoperative coaptation length for the large patch procedure was significantly greater than that with restrictive mitral annuloplasty $(P=.006)$ and the small patch repair $(P=.003$; Table 1).

For the posteromedial PM, the tethering force in the anterior strut chordae was significantly reduced postoperatively by all 3 repairs (Table 1). Compared with the FIMR condition, the force in the posterior intermediate chordae was moderately reduced by the small $(15.8 \% \pm 23.5 \%$ reduction) and large $(25.2 \% \pm 18.0 \%$ reduction) patch repairs. Moreover, the large patch repair significantly reduced the posterior intermediate chordal force compared with restrictive mitral annuloplasty $(3.7 \% \pm 23.9 \%$ reduction $)$.

For the anterolateral PM, the anterior strut chordal tethering force was significantly increased for both the small $(20.5 \% \pm 69.4 \%$ increase $)$ and the large $(11.8 \% \pm 27.2 \%$ increase $)$ patch treatments compared with FIMR. Restrictive mitral annuloplasty showed a moderate improvement $(13.0 \% \pm 19.1 \%$ reduction $)$ in the chordal force compared with FIMR. The tethering force in the posterior intermediate chordae originating from the

TABLE 1. Coaptation length and tethering forces for each chordae across all conditions

\begin{tabular}{lccccc}
\hline \multicolumn{1}{c}{ Variable } & Healthy & FIMR & RMA & Small patch & Large patch \\
\hline Sample size & 15 & 15 & 15 & 8 \\
Coaptation length (mm) & $4.8 \pm 0.5$ & $3.2 \pm 0.5^{*}$ & $4.7 \pm 0.6$ & $4.8 \pm 0.5$ & \\
Chordal force (N) & & & & \\
$\quad$ Posterior PM & & & & \\
$\quad$ Anterior strut & $0.71 \pm 0.31$ & $1.23 \pm 0.44 \ddagger$ & $0.89 \pm 0.33$ & $0.91 \pm 0.37$ \\
$\quad$ PIC & $0.28 \pm 0.13$ & $0.55 \pm 0.29 \S$ & $0.51 \pm 0.26$ & $0.39 \pm 0.18$ \\
Anterior PM & & & & $0.74 \pm 0.31$ \\
$\quad$ Anterior strut & $0.62 \pm 0.44$ & $0.80 \pm 0.52$ & $0.66 \pm 0.39$ & $0.78 \pm 0.43$ \\
$\quad$ PIC & $0.49 \pm 0.34$ & $0.71 \pm 0.51$ & $0.54 \pm 0.33$ & $0.46 \pm 0.24$ & $0.94 \pm 0.60$ \\
\hline
\end{tabular}

Statistical comparisons were made between experimental conditions for each quantitative measurement. FIMR, Functional ischemic mitral regurgitation; RMA, restrictive mitral annuloplasty; $P M$, papillary muscle; $P I C$, posterior intermediate chordae. *Significance of coaptation length between control and FIMR. †Significance of coaptation length between large patch and control. ఫ̦Significance between anterior strut chordal force from the anterior PM between FIMR and control. §Significance between posterior intermediate chordal force from the posterior PM between FIMR and control. 


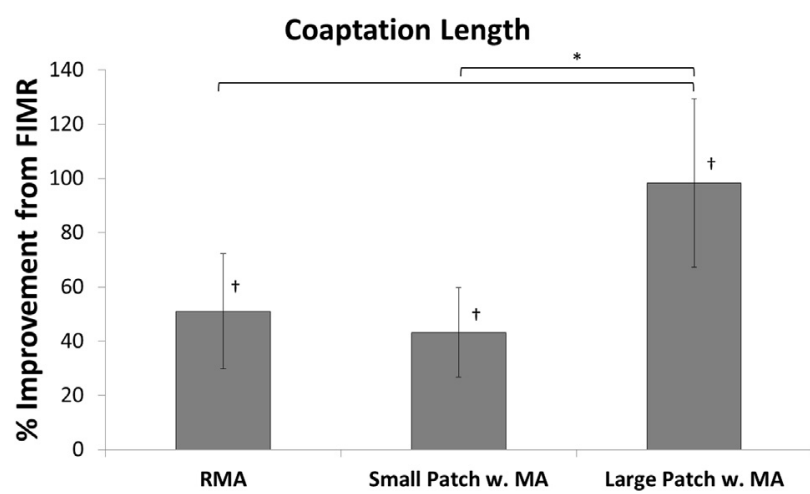

FIGURE 2. Improvement (percentage of increase) in coaptation length compared with functional ischemic mitral regurgitation $(F I M R)$ is shown for restrictive mitral annuloplasty (RMA) and small and large patch repairs. $M A$, Mitral annuloplasty. *Statistical significance between conditions $(P<.05)$. $\dagger$ Statistical significance between indicated condition and FIMR $(P<.05)$.

anterolateral PM was minimally affected by all the repair procedures (RMA, $4.7 \% \pm 48.4 \%$ reduction; small patch, $1.5 \% \pm 48.4 \%$ increase; large patch, $0.7 \% \pm 88.1 \%$ reduction) compared with FIMR.

\section{DISCUSSION}

Long-term postoperative repair success for restrictive mitral annuloplasty to treat FIMR has remained elusive..$^{7-10,15}$ Recent data have suggested preoperative patient characteristics that are predictive of repair failure. Of these, severely decreased coaptation ${ }^{16,17}$ and grossly enlarged ventricular dimensions ${ }^{6}$ are easily measured parameters indicative of a patient population that might benefit from an adjunct surgical procedure. Additionally, increased leaflet tethering ${ }^{7,10,15}$ after late ventricular remodeling is a mechanism of recurrent FIMR for restrictive mitral annuloplasty repair.

Thus, ALA with true-size mitral annuloplasty has emerged as an additive technique for FIMR. ${ }^{5,11}$ In the present study, ALA was performed in 2 sizes for comparison against restrictive mitral annuloplasty. All 3 repairs comparably alleviated FIMR, with no postoperative MR jets detected. Additionally, all 3 repairs restored coaptation to physiologic levels, with significant increases from the FIMR pathologic findings. Moreover, the large patch procedure resulted in an additional 1-mm gain in the coaptation length, because the large patch significantly increased the anterior leaflet surface area available for coaptation. Overall, this created additional redundancy to maintain a proper valvular seal, which has the potential to withstand late ventricular remodeling.

It was expected that the benefits of the extra coaptation length with the large patch would result in increased cyclic chordal forces owing to the larger exposed leaflet surface area. However, this was not observed for the anterior strut and posterior intermediate chordae originating from the posterior PM. In contrast, augmentation resulted in the best combined reduction in the tethering force for chordae originating from the displaced posterior PM. This most likely resulted from 2 mechanisms (Figure 4). First, the leaflet insertion points of the anterior strut chordae were displaced apically, toward the PMs, by the use of the patch. This effectively shorted the distance the chordae had to traverse between diastole and systole to maintain coaptation. Second, echocardiography showed the anterior strut chordae were moved into or near the coaptation zone. This site is known to experience relatively low chordal forces, because it is not actively engaged in resisting the transmitral pressure. ${ }^{18}$

The posterior intermediate chordae originating from the posterior PM also experienced a reduction in tethering forces. Three mechanisms were suspected (Figure 4). First, the posterior annulus was not hoisted directionally away from the pathologic PM, such as was done with restrictive annuloplasty. ${ }^{15}$ This effectively shortened the distance between the leaflet insertion point and the restraining PM, limiting extension of the chordae. Second, the increased anterior leaflet mobility reduced the overall loading of the posterior leaflet, such that the posterior leaflet did not traverse as far to oppose the anterior leaflet. This effectively limited elongation of the chordae from diastole to systole. Third, more of the posterior leaflet was involved with coaptation, effectively displacing the posterior intermediate chordae near to the coaptation zone, a site known to experience lower chordal forces.

From these experimental results, no measured change was seen in the posterior intermediate tethering forces for the restrictive mitral annuloplasty surgical repair. An increase in force was expected owing to the hoisting of the posterior annulus (Figure 4). However, it is likely this was offset by the creation of a functionally unicuspid valve, which resulted in a minimal posterior leaflet area exposed to the transmitral pressure gradient. Nonetheless, the posterior chordal forces remained elevated at pathologic levels.

Anterior strut chordae originating from the anterior PM did not benefit from ALA. Instead, a slight increase occurred in the tethering force for this chordal grouping. This suggests that significant PM displacement (such as was seen with the posterior PM) has a compensatory effect on the tethering forces in the presence of leaflet augmentation. Without PM displacement, the leaflet freely bellowed into the left atrium, further exposing the surface area to the transmitral pressure. In this setting, restrictive mitral annuloplasty resulted in a modest improvement in the tethering force, which contributed to the smaller mitral orifice.

None of the repairs had an effect on the posterior intermediate chordae originating from the anterior PM. Without significant PM displacement, hoisting of the 


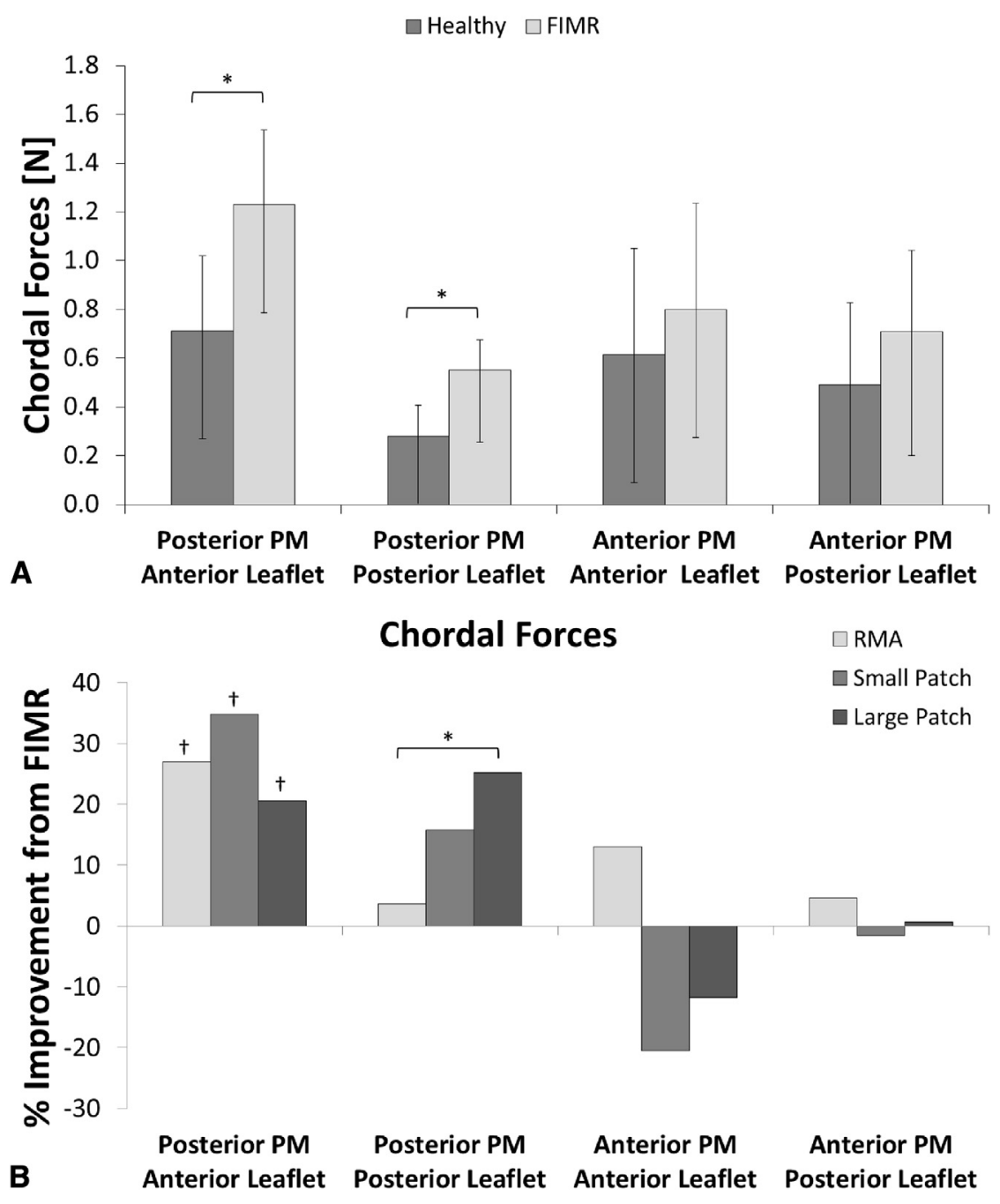

FIGURE 3. A, Chordal forces are shown for all 4 chordae in healthy and functional ischemic mitral regurgitation (FIMR) conditions. B, Improvement (percentage of reduction) in chordal force compared with FIMR is shown for all 4 chordae for restrictive mitral annuloplasty $(R M A)$ and small and large patch repairs. *Statistical significance between conditions $(P<.05)$. $\dagger$ Statistical significance between indicated condition and FIMR $(P<.05) . P M$, Papillary muscle.

posterior annulus did not exacerbate posterior leaflet tethering. The leaflet remained mobile and spanned more of the mitral orifice to oppose the anterior leaflet.

\section{Study Limitations}

Although the in vitro model used in the present study simulated physiologic function, hemodynamics, and the mechanics of a native mitral valve, the rigid ventricle did not mimic the dynamic annular and ventricular motion. However, mitral annuloplasty, as performed in the repair conditions, is known to reduce annular dynamics. ${ }^{19}$ Although ventricular wall motion was not simulated, the distance between the PMs and the annular plane has been shown to remain relatively constant throughout the cardiac cycle. ${ }^{20}$ Moreover, relative PM displacements were informed from the clinical data, and the left heart simulator has been shown to mimic the systolic function and mechanics of an established chronic FIMR ovine model. $^{12}$

\section{CONCLUSIONS}

The results of the present study demonstrated that in the setting of FIMR due to severe LV geometric distortion, ALA is a possible surgical option within the limitations of the study design. The data from each patch size suggested that the augmentation dimensions do not need to be precisely tailored because sufficient repair is achievable within a range. An oversized patch can be targeted because it provided the best gains in coaptation length and reduction in chordal tethering. Although all repairs abolished MR acutely, the repairs that create the greatest coaptation could conceivably produce a more robust and lasting repair in the chronic stage. However, clinically, the need still exists to best identify which patients with altered MV geometries 
FIMR

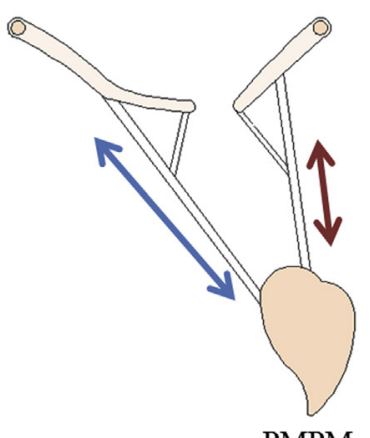

PMPM
RMA

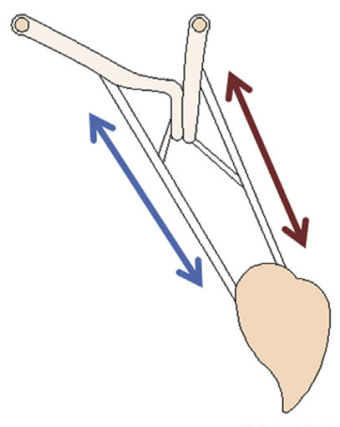

PMPM
ALA

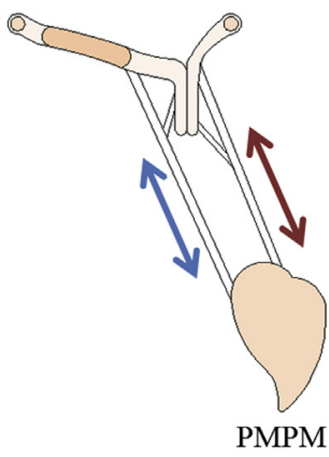

\section{$\longrightarrow$ Anterior Leaflet Strut Chordal Tethering Magnitude Posterior Leaflet Intermediate Chordal Tethering Magnitude}

FIGURE 4. Schematic representation of the mechanisms that affect the chordal forces in the functional ischemic mitral regurgitation (FIMR) and repair conditions. Left, Chordal tethering is a result of annular dilatation and papillary muscle (PM) displacement and pressure forces. Middle, Posterior tethering force is a balance between the pressure force and the opposing displacements of the papillary muscles and annulus reshaping. Right, Anterior strut tethering is reduced by displacing the chordal insertion apically and into the coaptation zone. RMA, Restrictive mitral annuloplasty; $A L A$, anterior leaflet augmentation; PMPM, posteromedial papillary muscle.

would most benefit from an adjunct procedure compared with simple restrictive mitral annuloplasty.

We would like to acknowledge the contributions of Steven A. Touchton, Jr, Eric L. Pierce, and Joan Fernandez Esmerates.

\section{References}

1. Tibayan FA, Rodriquez F, Zasio MK, Bailey L, Liang D, Daughters GT, et al. Geometric distortions of the mitral valvular-ventricular complex in chronic ischemic mitral regurgitation. Circulation. 2003;108:II-116-21.

2. Grigioni F, Enriquez-Sarano M, Zehr KJ, Bailey KR, Tajik J. Ischemic mitral regurgitation: long-term outcome and prognostic implications with quantitative Doppler assessment. Circulation. 2001;103:1759-64.

3. Bax JJ, Braun J, Soner ST, Klautz R, Holman ER, Versteegh MI, et al. Restrictive annuloplasty and coronary revascularization in ischemic mitral regurgitation results in reverse left ventricular remodeling. Circulation. 2004;110(11 Suppl 1): II-103-8.

4. Kron IL, Green GR, Cope JT. Surgical relocation of the posterior papillary muscle in chronic ischemic mitral regurgitation. Ann Thorac Surg. 2002;74:600-1.

5. Hines MH, Kincaid EH, Cook AL, Kon ND. Anterior leaflet augmentation for ischemic mitral regurgitation in patients with anomalous left coronary artery from the pulmonary artery and preserved left ventricular function. J Thorac Cardiovasc Surg. 2011;142:716-8.

6. Braun J, Bax JJ, Versteegh MI, Voight PG, Holman ER, Klautz RJ, et al. Preoperative left ventricular dimensions predict reverse remodeling following restrictive mitral annuloplasty in ischemic mitral regurgitation. Eur J Cardiothorac Surg. 2005;27:847-53.

7. Hung J, Papakostas L, Tahta SA, Hardy BG, Bollen BA, Duran CM, et al. Mechanism of recurrent ischemic mitral regurgitation after annuloplasty: continued LV remodeling as a moving target. Circulation. 2004;110(11 Suppl 1):II-85-90.

8. Matsunaga A, Tahta SA, Duran CMG. Failure of reduction annuloplasty for functional ischemic mitral regurgitation. J Heart Valve Dis. 2004;13:390-7.

9. McGee EC, Gillinov AM, Blackstone EH, Rajeswaran J, Cohen G, Najam F, et al. Recurrent mitral regurgitation after annuloplasty for functional ischemic mitral regurgitation. J Thorac Cardiovasc Surg. 2004;128:916-24.
10. Kuwahara E, Otsuji Y, Iquro Y, Ueno T, Zhu F, Mizukami N, et al. Mechanism of recurrent/persistent ischemic/functional mitral regurgitation in the chronic phase after surgical annuloplasty: importance of augmented posterior leaflet tethering. Circulation. 2006;114(1 Suppl):I-529-34.

11. Kincaid EH, Riley RD, Hines MH, Hammon JW, Kon ND. Anterior leaflet augmentation for ischemic mitral regurgitation. Ann Thorac Surg. 2004;78: 564-8.

12. Siefert AW, Rabbah JP, Koomalsingh KJ, Touchton SA Jr, Saikrishnan N, McGarvey JR, et al. In vitro mitral valve simulator mimics systolic valvular function of chronic ischemic mitral regurgitation ovine model. Ann Thorac Surg. 2013;95:825-30.

13. Nielsen SL, Soerensen DD, Libergren P, Yoganathan AP, Nygaard H. Miniature C-shaped transducers for chordae tendineae force measurements. Ann Biomed Eng. 2004;32:1050-7.

14. Jimenez J, Soerensen DD, He Z, He S, Yoganathan AP. Effects of a saddle shaped annulus on mitral valve function and chordal force distribution: an in vitro study. Ann Biomed Eng. 2003;31:1171-81.

15. Zhu F, Otsuji Y, Yotsumoto G, Yuasa T, Ueno T, Yu B, et al. Mechanism of persistent ischemic mitral regurgitation after annuloplasty: importance of augmented posterior mitral leaflet tethering. Circulation. 2005;112(9 Suppl): I-396-401.

16. Kongsaerepong V, Shiota M, Gillinov AM, Song J-M, Fukuda S, McCarthy PM, et al. Echocardiographic predictors of successful versus unsuccessful mitral valve repair in ischemic mitral regurgitation. Am J Cardiol. 2006;98:504-8.

17. Roshanali F, Mandegar MH, Yousefnia MA, Rayatzadeh H, Alaeddini F. A prospective study of predicting factors in ischemic mitral regurgitation recurrence after ring annuloplasty. Ann Thorac Surg. 2007;84:745-9.

18. Jimenez JH, Soerensen DD, He Z, Ritchie J, Yoganathan AP. Effects of papillary muscle position on chordal force distribution: an in-vitro study. J Heart Valve Dis. 2005; 14:295-302.

19. Rausch MK, Bothe W, Kvitting JP, Swanson JC, Miller DC, Kuhl E. Mitral valve annuloplasty: a quantitative clinical and mechanical comparison of different annuloplasty devices. Ann Biomed Eng. 2012;40:750-61.

20. Joudinaud TM, Kegel CL, Flecher EM, Weber PA, Lansac E, Hvass U, et al. The papillary muscles as shock absorbers of the mitral valve complex: an experimental study. Eur J Cardiothorac Surg. 2007;32:96-101. 